\title{
Estudo da síndrome de tração macular vítreo-retiniana idiopática por meio da tomografia de coerência óptica: relato de casos
}

\author{
A study of idiophatic vitreomacular traction syndrome with optical coherence \\ tomography images:cases report
}

\author{
Hélio Paulo Primiano Júnior ${ }^{1}$ \\ Aloísio Fumio Nakashima ${ }^{2}$ \\ Otacílio Oliveira Maia Júnior ${ }^{3}$ \\ Maria Teresa Brizzi Bonanomi ${ }^{4}$ \\ Yoshitaka Nakashima $^{5}$
}

\begin{tabular}{|l|}
\hline RESUMO \\
\hline Objetivos: Avaliar alterações estruturais observadas na síndrome de \\
tração macular vítreo-retiniana idiopática (STMI) pela tomografia de \\
coerência óptica (OCT), antes e depois da vitrectomia; identificar si- \\
tuações em que a tomografia de coerência óptica fornece informações \\
adicionais da morfologia e patogênese desta doença. Métodos: Imagens \\
tomográficas (OCT Stratus) comparativas da estrutura vítreo-retiniana \\
foram realizadas em quatro pacientes, em estágios variados da síndrome \\
de tração macular vítreo-retiniana idiopática. Resultados: A tomografia \\
de coerência óptica Stratus apresenta imagens vítreo-retinianas que \\
auxiliam na conduta e tratamento da síndrome de tração macular vítreo- \\
retiniana idiopatica. Conclusão: O exame da tomografia de coerência \\
óptica permite boa visualização da arquitetura morfológica do vítreo e da \\
retina na síndrome de tração macular vítreo-retiniana idiopática. Este \\
exame nos auxilia na compreensão da patogênese, no planejamento do \\
tratamento, clínico ou cirúrgico, e na avaliação pós-operatória das \\
alterações estruturais retinianas.
\end{tabular}

Descritores: Tomografia de coerência óptica; Descolamento do vítreo; Vitrectomia; Triancinolona; Edema macular cistóide

Médico Colaborador do Departamento de Oftalmologia do Hospital das Clínicas da Faculdade de Medicina da Universidade de São Paulo - USP - São Paulo (SP) - Brasil.

Acadêmico da Faculdade de Medicina da USP - São Paulo (SP) - Brasil

${ }^{3}$ Pós-Graduando (Nível Doutorado) do Departamento de Oftalmologia do Hospital das Clínicas da Faculdade de Medicina da USP - São Paulo (SP) - Brasil.

${ }^{4}$ Médica Assistente Doutora do Departamento de Oftalmologia do Hospital das Clínicas da Faculdade de Medicina da USP - São Paulo (SP) - Brasil.

${ }_{5}^{5}$ Médico Assistente Doutor do Departamento de Oftalmologia do Hospital das Clínicas da Faculdade de Medicina da USP - São Paulo (SP) - Brasil.

Endereço para correspondência: Hélio Paulo Primiano Júnior. Rua Henrique Lupo, 777 - Araraquara (SP) CEP 14802-440

E-mail: helioprimiano@hotmail.com

Recebido para publicação em 04.05.2005

Última versão recebida em 12.06.2006

Aprovação em 26.06.2006

Nota Editorial: Depois de concluída a análise do artigo sob sigilo editorial e com a anuência do Dr. Arnaldo Furman Bordon sobre a divulgação de seu nome como revisor, agradecemos sua participação neste processo.

\footnotetext{
$\frac{\text { INTRODUÇÃOO }}{\text { A síndrome de tração vitreomacular idiopática (STMI) é uma doença }}$ incomum, de etiologia desconhecida, que acomete a interface vítreo-retiniana. Nestes casos, o descolamento incompleto do vítreo posterior induz alterações estruturais e funcionais retinianas secundárias à força de tração centrípeta vítrea ${ }^{(1-3)}$.

Na STMI, opacidades anormais estão presentes no vítreo ao redor da região macular. Acredita-se que a sua origem esteja relacionada à proliferação induzida pelo descolamento parcial do vítreo posterior associado à tração na mácula, no nervo óptico, assim como nas membranas epiretinianas. A região macular pode tornar-se distorcida, cística ou em tenda, com pequeno descolamento de retina tracional ${ }^{(2,4)}$.

A vitrectomia poderá ser considerada se o paciente apresentar baixa da acuidade visual significativa. A separação entre o vítreo posterior e a retina, na área de adesão anormal, pode ocorrer espontaneamente ${ }^{(5-7)}$.

A vitrectomia via pars plana pode ser associada à remoção da membrana limitante interna. O tratamento cirúrgico é efetivo na remoção da aderência na interface vitreomacular, restaurando a anatomia macular e levando à
} 
regressão do edema macular cistóide e a melhora na acuidade visual $^{(8-9)}$.

A tomografia de coerência óptica (OCT) possibilita a avaliação da interface vítreo-retiniana da síndrome de tração macular $^{(10)}$. Após a intervenção cirúrgica nos casos de STMI, a remoção da tração vitreomacular leva a uma lenta reorganização tecidual ${ }^{(9)}$.

\section{RELATO DE CASOS}

\section{Caso 1}

DMCP, 57 anos, sexo feminino, branca, natural de Araraquara (SP), sem alterações sistêmicas ou oculares prévias. Apresentava acuidade visual (AV) corrigida de 20/20 e 20/30-3, respectivamente, no olho direito (OD) e no olho esquerdo (OE). Ao exame de motricidade ocular, reflexos fotomotores e biomicroscopia de segmento anterior, não apresentava alterações. Na fundoscopia apresentava pequeno espessamento foveal no $\mathrm{OE}$. Na angiofluoresceinografia (AF) apresentava hiperfluorescência por extravazamento progressivo de contraste, na região foveal do OE. Ao exame de OCT observou-se aderência da hialóide com a formação de imagem "em tenda", com ausência da depressão foveal (Figura 1). Optou-se por conduta expectante já que esta alteração localizava-se na região superficial da retina neurosensorial, poupando a região de fotorreceptores, e a baixa acuidade visual não era significativa para o paciente. Após 3 meses de evolução observou-se separação da tração e remodelação da depressão foveal detectada através do OCT.

\section{Caso 2}

UH, 65 anos, sexo feminino, branca, com história de baixa de AV progressiva no OD há 6 meses. A paciente não apresentava antecedentes pessoais e familiar de doenças sistêmicas ou oculares. Ao exame ocular, a AV, com a melhor correção óptica era de 20/400 no OD e 20/25 no OE. O exame de fundoscopia do OD demonstrava uma desorganização macular com tração vítreoretiniana. A AF apresentava hiperfluorescência com progressivo extravazamento de corante que se acumulava na região foveal do

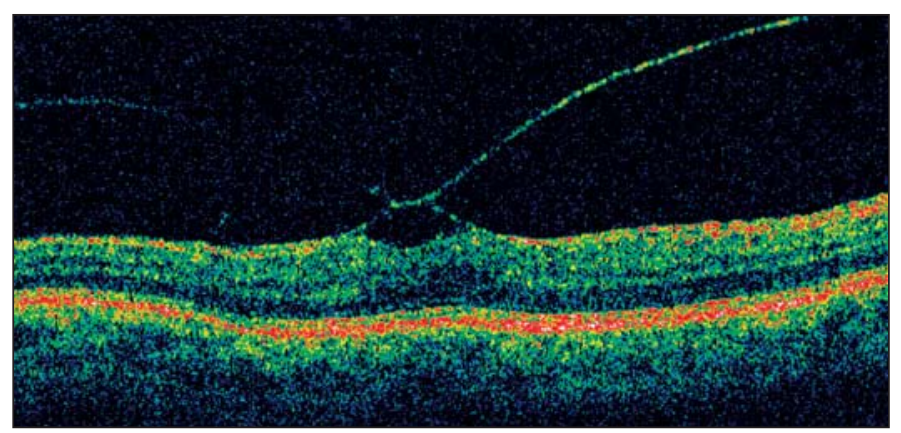

Figura 1 - "Sinal da águia" (Caso 1). Imagem de OCT Stratus do OE, mostrando a área de aderência vítreo-retiniana na região macular, com ausência da depressão foveal.
OD. O OCT do OD apresentava descolamento parcial de vítreo posterior desorganizando a estrutura da fóvea, com aspecto de tenda ("sinal da cordilheira") e formação de descolamento tracional localizado da retina (Figura 2).

$\mathrm{Na}$ estrutura interna da retina neurosensorial observavamse imagens de baixa refletividade, arredondadas, sugerindo edema macular.

Foi realizada vitrectomia com descolamento do vítreo posterior, ao redor do nervo óptico e em região macular, com troca fluido gasosa e troca ar-gás (C3F8 a 14\%).

Após 15 dias a paciente apresentava visão de 20/200 e imagem do OCT mostrando ausência de tração vítrea, mantendo, no entanto, a área de formação cística com baixa refletividade intra-retiniana na região foveal. Não se observava a depressão foveal. Com 2 meses de pós-operatório, a paciente apresentava AV de 20/150 e OCT diminuição das cavidades císticas intra-retinianas ao OCT.

Com 4 meses de pós-operatório, a AV mantinha-se em 20/150 no olho operado que esboçava início de remodelação da depressão foveal ao OCT (Figura 3). Procedeu-se à injeção de $4 \mathrm{mg}$ de triancinolona intravítrea com reorganização total da depressão foveal ao OCT em 1 semana. As medidas de pressão intra-ocular se mantiveram entre 13 e 16 mmHg. Após 3 meses da injeção intravítrea, a imagem tomográfica mostrava a depressão foveal sem cistos apesar de a visão manter-se em 20/150.

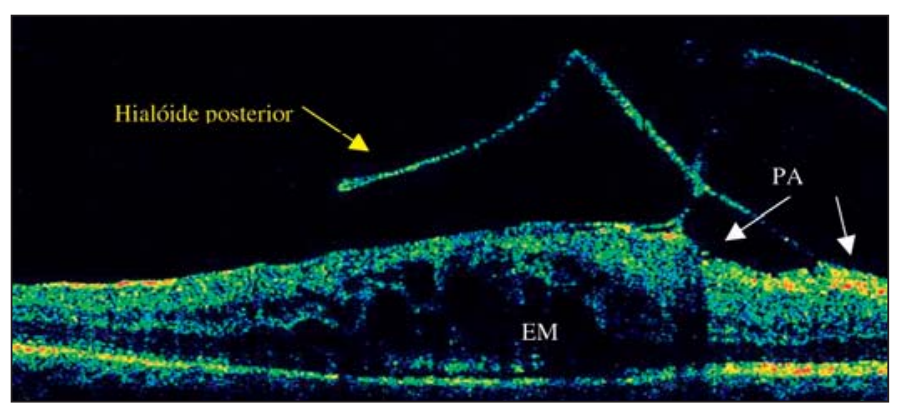

Figura 2 - "Sinal da cordilheira" (Caso 2). Imagem do OCT Stratus do OD do caso 2 mostrando pontos de aderência da hialóide posterior na retina neurossenssorial (PA - setas brancas) e edema macular (EM). Linha tracejada representando a altura da "tenda" (623 $\mu \mathrm{m})$.

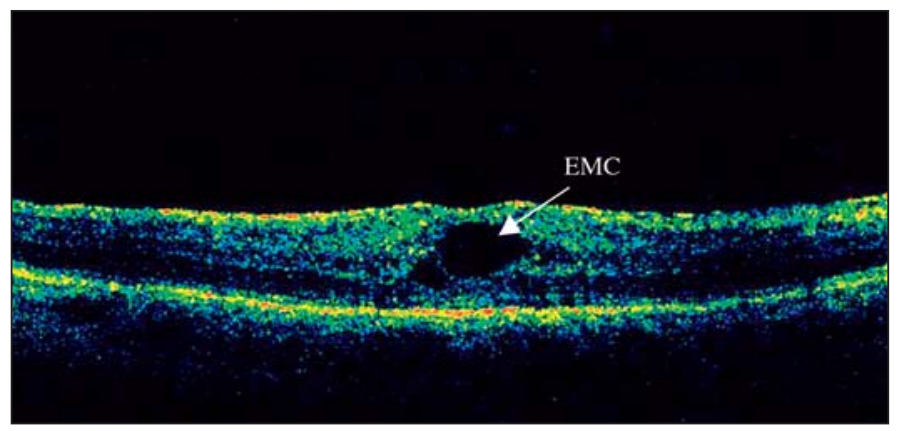

Figura 3 - "Cavidade cística” (Caso 2). Imagem do OCT Stratus do OD do caso 4, no $4^{\circ}$ mês de pós-operatório, com início de formação da depressão foveal e persistência dos cistos intra-retinianos. 


\section{Caso 3}

NRF, 68 anos, sexo feminino, branca, aposentada, natural e procedente de São Paulo apresentando baixa de AV progressiva há aproximadamente 1 ano e meio no $\mathrm{OD}$, sem antecedentes pessoais de cirurgia oftalmológica e doenças oculares ou sistêmicas.

A paciente apresentava AV com correção óptica de 20/150 e 20/25, respectivamente, no OD e no OE. Ao exame fundoscópico apresentava, no OD, tortuosidade de vasos da arcada temporal superior e inferior com condensação vítrea na região foveal. O exame de OCT deste olho apresentava imagem de tração vitreomacular, por descolamento parcial de vítreo posterior, desorganizando a estrutura da fóvea e formando aspecto de "tenda", associada presença de membrana de alta refletividade, gerando moderada tração centrípeta da superfície retiniana. Ainda neste exame, observavam-se áreas arredondadas de baixa refletividade interna em meio a camadas correspondentes à retina neurosensorial, sugerindo a presença de edema cístico intra-retiniano.

Foi realizada vitrectomia com descolamento total do vítreo e remoção de membrana epirretiniana macular. Na primeira semana de pós-operatório a paciente referia melhora da metamorfopsia. Ao exame, o olho operado apresentava discreta reação inflamatória, e no OCT visualizava-se retina espessada, sem sinais que sugerissem a presença de tração vítreo-retiniana. A AV corrigida no olho operado manteve-se 20/150.

Com 1 mês de pós-operatório, a AV era de 20/80, e a imagem do OCT apresentava perfil plano da retina, inclusive na área compatível com a fóvea, associada à manutenção dos cistos intra-retinianos.

Apenas com 2 meses de pós-operatório, a fóvea começou a apresentar, ao OCT, um esboço de remodelação da sua depressão e diminuição das cavidades císticas de baixa refletividade interna compatíveis com a absorção do líquido subretiniano mantendo a $\mathrm{AV}$ em 20/70.
Os exames foram sendo realizados mensalmente com OCT, porém a imagem sugestiva de reabsorção total do edema cístico e de desenho compatível com o reposicionamento da depressão foveal foi observada apenas no $7^{\circ}$ mês de pós-operatório quando a $\mathrm{AV}$ era de $20 / 50^{-3}$. Esta visão manteve-se até o último exame, realizado no $11^{\circ}$ mês de seguimento após a cirurgia.

\section{Caso 4}

JPO, 65 anos, masculino, natural e procedente de São José dos Campos, aposentado. Paciente com queixa de baixa AV progressiva no $\mathrm{OE}$ há 4 meses. Ao exame apresentava AV no OD e no OE, respectivamente, de 20/20 e 20/200, e fundoscopia com áreas de condensação vítrea na região ao redor do nervo óptico e na fóvea do OE, sugerindo a presença de condensação vítrea nestas regiões (Figura 4A). Na AF observavam-se hiperfluorescência progressiva na região papilar (Figura 4B) e discreta hiperfluorescência progressiva na região justafoveal na fase de recirculação do $\mathrm{OE}$, sugerindo a presença de edema macular.

O OCT de OE apresentava imagem de aderência da hialóide na mácula e no nervo óptico, tracionando o nervo óptico e a região macular, originando o descolamento de retina tracional e a distorção da arquitetura peri-papilar. A descrição da imagem tomográfica simula uma "corcova de camelo" (Figura 5A).

Realizou-se cirurgia de vitrectomia via pars plana com descolamento de vítreo posterior, troca fluido-gasosa e troca argás (C3F8 a 14\%).

Já no $15^{\circ}$ dia de pós-operatório, o paciente apresentava melhora da AV para 20/80 e, após 2 meses e meio, apresentava AV de 20/40 em AO. As imagens do OCT apresentavam restituição dos níveis normais de refletividade interna das camadas mais profundas da retina neurosensorial, mostrando resolução do descolamento tracional e do edema (Figura 5B). A depressão foveal estava presente.
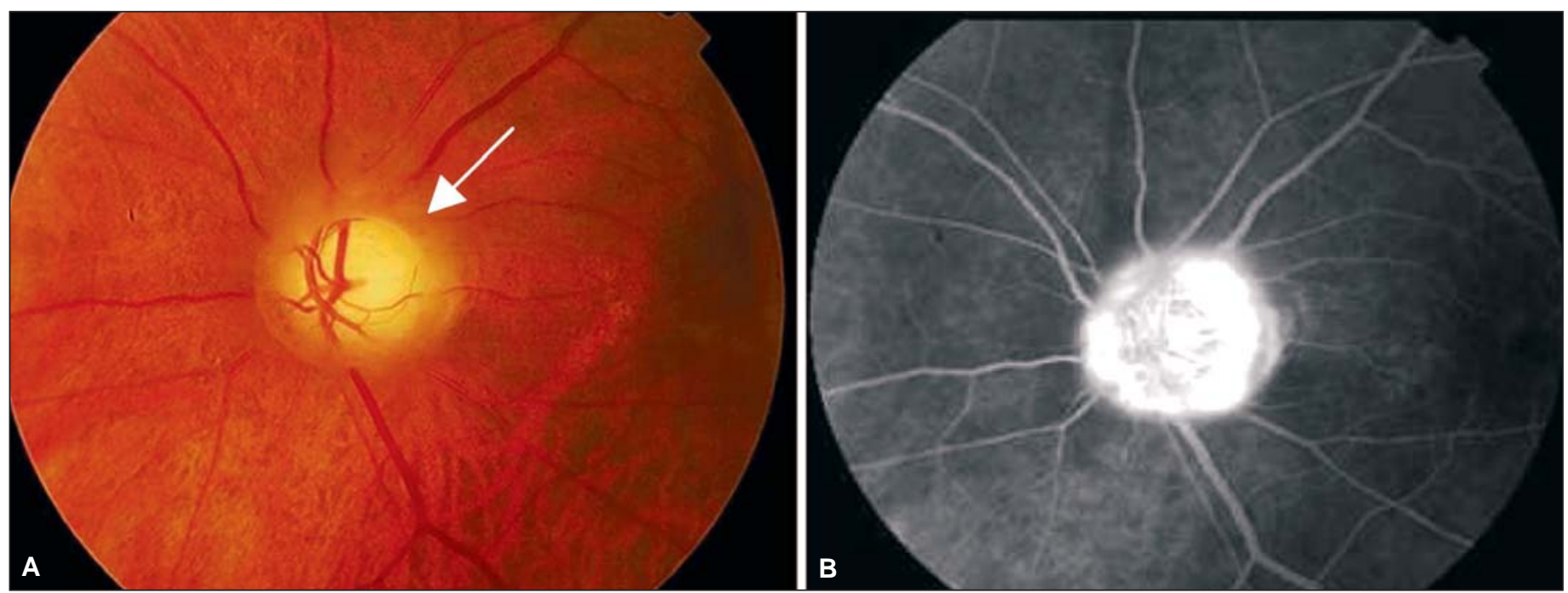

Figura 4 - (Caso 4). A: retinografia mostrando condensação vítrea ao redor do disco óptico (seta); B: angiofluresceinografia na fase de recirculação evidenciando hiperfluorescência progressiva durante o exame ao redor do disco óptico. 

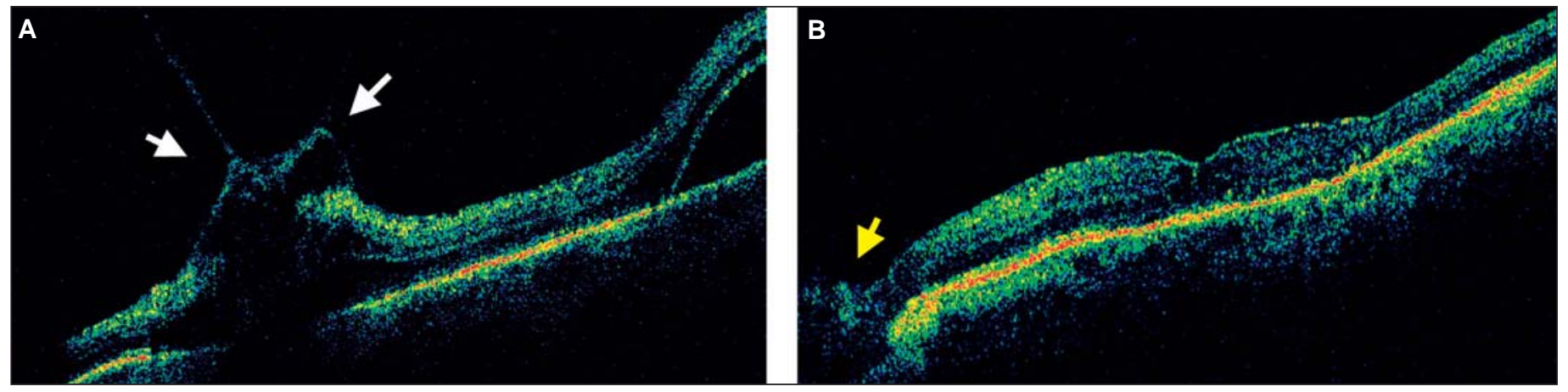

Figura 5 - (Caso 4) "Sinal da corcova de camelo". A: Imagem OCT Stratus do OE mostrando sinais de alta refletividade (setas brancas) na margem do nervo óptico, compatíveis com áreas de tração, exercendo força de tração centrípeta, assim como na região macular, sugerindo o aparecimento de descolamento tracional de retina. B: Imagem do OCT Stratus, no pós-operatório, com scan de $10 \mathrm{~mm}$ de comprimento, apresentando ausência dos sinais de alta refletividade compatíveis com áreas de tração, tanto na margem do nervo óptico (seta amarela) quanto na superfície retiniana, sugerindo o restabelecimento da arquitetura morfológica do nervo óptico e da retina.

\section{DISCUSS ÃO}

A síndrome de tração macular vem sendo descrita como uma alteração na interface vítreo-retiniana, onde a presença de miofibroblastos, associados ou não a camadas de colágeno, impede que haja um descolamento completo entre o vítreo posterior e a retina ${ }^{(1-11)}$.

A ultraestrutura da interface vítreo-retiniana apresenta dois padrões básicos: camada celular aderida à membrana limitante interna, e proliferação fibrocelular separada da membrana limitante interna por uma camada de colágeno, semelhante às da membrana epirretiniana idiopática. Em ambos os grupos o tipo celular predominante é o miofibroblasto, o que explica a alta prevalência de edema macular cistóide e a progressiva tração vitreomacular( ${ }^{(3)}$. A angiografia fluoresceínica pode demonstrar extravazamento do corante nas regiões da macular e peri-papilar ${ }^{(1)}$.

As alterações estruturais da retina decorrente destas alterações visualizadas ao exame de OCT têm complementado o diagnóstico e auxiliado na conduta, expectante ou não, a ser adotada.

Desta forma descrevemos alguns dos sinais visualizados ao exame de tomografia de coerência óptica em pacientes com síndrome de tração macular:

1) Sinal da águia: Tração retiniana por aderência da hialóide posterior, com o aspecto de pequenas tendas, distorcendo a anatomia macular, com ausência de depressão foveal, simulando uma "águia de asas abertas" (Figura 1), e caracterizando estágios iniciais de tração vítreo-retiniana.

2) Sinal da cordilheira: Áreas de tração retiniana por aderência da hialóide posterior, com aspecto de altas tendas, simulando uma "cordilheira". A tração na retina neuro-sensorial possibilita o aparecimento de alterações na refletividade interna de suas camadas mais externas, onde podem ser observadas áreas de baixa refletividade, difusas ou císticas, porém sem o aspecto de descolamento tracional de retina neurosensorial (Figura 2).
3) Sinal da corcova de camelo: Tração de nervo óptico, por aderência da hialóide posterior associado a descolamento tracional de retina, sugerindo o aspecto de "corcova de camelo". Este aspecto se deve à tração da hialóide, tanto na região do nervo óptico quanto na retina neurosensorial. Observa-se distorção da anatomia do nervo óptico, assim como da retina, devido à tração vítreo-retiniana. Alterações de baixa refletividade interna também podem ser observadas semelhante aos casos de estágio 2 (Figura 5B).

4) Cavidade cística: Presença de área de hiporrefletividade, circunscrita, localizada na região compatível com a retina neurosensorial, sugestiva de cisto intra-retiniano, que pode ser visualizada no pós-operatório destes pacientes.

A visualização de achados tomográficos descritos acima, em associação com os exames oftalmológico e angiográfico, tem proporcionado uma maior segurança na conduta da STMI. Acreditamos que alguns fatores devem ser analisados antes de ser direcionada a conduta, dos quais destacamos abaixo: distorção da arquitetura tecidual por tempo prolongado, originando edema localizado e disfunção progressiva do tecido retiniano; a presença de intensa hiporrefletividade nas camadas mais profundas da retina neurosensorial, onde se localizam os fotorreceptores; e presença de descolamento tracional macular.

Assim, casos em que apresentam boa acuidade visual e pequena distorção da arquitetura retiniana apresentam melhores prognósticos. Estes casos podem ser acompanhados apenas sob observação clínica, sabendo que a separação entre o vítreo e a retina, na região de adesão anormal e tração, pode ocorrer espontaneamente ${ }^{(5-7)}$.

A opção pela vitrectomia posterior tem sido aventada aos primeiros sinais de piora prognóstica. Sinais semiológicos de piora da metamorfopsia e baixa progressiva da acuidade visual, associados a imagens fundoscópicas e tomográficas que retratam distorções na arquitetura retiniana, como visualizados nos sinais da "cordilheira" e de "corcova de camelo", podem sugerir a intervenção.

Em meio ao procedimento cirúrgico de vitrectomia, a injeção de triancinolona intravítrea intra-operatória tem auxiliado na 
visualização da hialóide posterior no ato cirúrgico bem como na resolução dos edemas residuais no pós-operatório ${ }^{(11)}$.

A realização da troca fluido-gasosa e ar-gás pode ser benéfica no ato cirúrgico e no pós-operatório. A força de tração vitreoretiniana nesta síndrome é um fator importante a ser considerado, quando imaginamos que durante a cirurgia, a remoção da tração ocorre mecanicamente com o auxílio de pinças. Durante o processo de descolamento do vítreo posterior pode-se tracionar a retina e originar buracos maculares ou lamelares devido à forte aderência intermediada por miofibroblastos. Assim, a presença do gás teria a função de tamponamento destes buracos maculares, muitas vezes não detectados durante o procedimento.

Entretanto não há um consenso entre os cirurgiões, quanto à realização de troca ar-gás ${ }^{(10-11)}$. Neste estudo, optamos pela realização de troca ar-gás em todos os pacientes, acreditando que este procedimento contribuiria para o melhor prognóstico visual pós-operatório.

A presença de edema cístico de retina no pós-operatório se apresentou como um fator de mau prognóstico visual, mesmo após o seu tratamento com drogas esteroidais e/ou não esteroidais, tópicas ou intravítreas.

A melhora anatômica pós-operatória nem sempre é acompanhada de melhora na acuidade visual, refletindo prováveis alterações funcionais e microestruturais retinianas, que podem não se restabelecer após a intervenção cirúrgica. Entretanto, lembramos que, comumente observa-se melhora pósoperatória dos sintomas de metamorfopsia, uma das principais queixas destes pacientes.

\section{CONCLUSÃO}

O OCT é uma arma semiológica indispensável no diagnóstico e tratamento da STMI. Suas imagens apresentam boa definição no estudo, inicialmente, da desorganização estrutural vítreo-retiniana e, posteriormente, no acompanhamento da reformulação da anatomia normal após resolução do processo tracional.

\section{ABSTRACT}

Purpose: To evaluate the features of idiophatic vitreomacular traction syndrome (VMT) with ocular coherence tomography
(OCT - Stratus), before and after vitrectomy; to identify situations where ocular coherence tomography provides additional information on disease morphology and pathogenesis. Methods: Comparative retinal ocular coherence tomography imaging was performed in four patients with different stages of vitreomacular traction syndrome. Results: Standard-resolution ocular coherence tomography Stratus provides vitreousretinal images that help the management and treatment of vitreomacular traction syndrome. Conclusions: Standard-resolution ocular coherence tomography allows good visualization of the architectural morphology of the vitreous and retina in vitreomacular traction syndrome. It helps understanding its pathogenesis, planning its treatment, clinical or surgical, and evaluating retinal structural alterations after surgery.

Keywords: Tomography, optical coherence; Vitreous detachment; Vitrectomy; Triamcinolone; Macular edema, cystoid

\section{REFERÊNCIAS}

1. Hikichi T, Yoshida A, Trempe CL. Course of vitreomacular traction syndrome. Am J Ophthalmol. 1995;119(1):55-61.

2. Smiddy WE, Green WR, Michels RG, de la Cruz Z. Ultrastructural studies of vitreomacular traction syndrome. Am J Ophthalmol. 1989;107(2):177-85.

3. Gandorfer A, Rohleder M, Kampik A. Epiretinal pathology of vitreomacular traction syndrome. Br J Ophthalmol. 2002;86(8):902-9.

4. Yamada N, Kishi S. Tomographic features and surgical outcomes of vitreomacular traction syndrome. Am J Ophthalmol. 2005;139(1):112-7. Comment in: Am J Ophthalmol. 2005;140(4):765-6; author reply 766.

5. McDonald HR, Johnson RN, Schatz H. Surgical results in the vitreomacular traction syndrome. Ophthalmology. 1994;101(8):1397-402; discussion 1403.

6. Kusaka S, Saito Y, Okada AA, Sasamoto M, Hayashi A, Ohji M, Tano Y. Optical coherence tomography in spontaneously resolving vitreomacular traction syndrome. Ophthalmologica. 2001;215(2):139-41.

7. Carpineto P, Ciancaglini M, Aharrh-Gnama A, Agnifili L, Mastropasqua L. Optical coherence tomography and retinal thickness analyzer features of spontaneous resolution of vitreomacular traction syndrome: a case report. Eur J Ophthalmol. 2004;14(1):67-70.

8. Petropoulos IK, Stangos AA, Brozou CG, Kapetanios AD, Pournaras CJ. [Vitrectomy for vitreo-macular traction syndrome] Klin Monatsbl Augenheilkd. 2003;220(3):122-6. French.

9. Uchino E, Uemura A, Doi N, Ohba N. Postsurgical evaluation of idiopathic vitreomacular traction syndrome by optical coherence tomography. Am J Ophthalmol. 2001;132(1):122-3.

10. Larsson J. Vitrectomy in vitreomacular traction syndrome evaluated by ocular coherence tomography (OCT) retinal mapping. Acta Ophthalmol Scand. 2004;82(6):691-4.

11. Doi N, Uemura A, Nakao K, Sakamoto T. Vitreomacular adhesion and the defect in posterior vitreous cortex visualized by triamcinolone-assisted vitrectomy. Retina. 2005;25(6):742-5.

\section{Visite a site eletrônico dos ABD www.aboonline.com.br}

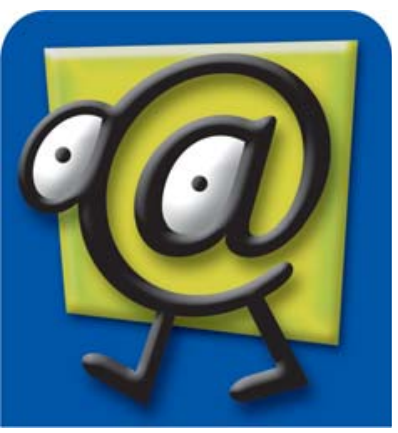

